Logos. Anales del Seminario de Metafísica

ISSN: 1575-6866

http://dx.doi.org/10.5209/asem.70836

\title{
Transhumanismo y neuroeducación en perspectiva orteguiana ${ }^{1}$
}

\author{
Javier Gracia-Calandín²
}

Recibido: 14 de octubre de 2019 / Aceptado: 19 de marzo de 2020

Resumen. Este artículo incide en la significatividad de los fines de la educación y la importancia vital de los deseos. Frente a las pretensiones de una tecnología a merced del mercado de las promesas transhumanistas, se retoma la pedagogía del cascabel de Ortega y la importancia de cultivar deseos ascendentes, que no tienen su fundamento en la técnica sino en la vida humana (y no posthumana). El artículo concluye con una defensa del compromiso ético-cívico de una genuina educación humanista radicada en el sentimiento moral de la compasión.

Palabras clave: tanshumanismo; neuroeducación; moral; mejoramiento; José Ortega y Gasset.

\section{[en] Transhumanism and neuroeducation in Ortega perspective}

\begin{abstract}
The aim of this paper is to emphasize the significance of moral education and the vital importance of desires. Faced with the pretensions of a transhumanist market-based technology, it is considered the importance of cultivating ascending desires and Jingle bell pedagogy of José Ortega y Gasset, which are based on human (and not post-human) life. The article concludes with a defense of the civic ethical commitment of a genuine humanistic education based on the moral feeling of compassion. Key words: transhumanism; neuroeducation; moral; enhancement; José Ortega y Gasset.
\end{abstract}

Sumario: 1. Introducción. Neuroeducación y transhumanismo, temas de nuestro tiempo; 2 . Hipertrofia de la técnica y crisis de los deseos; 2.1. Haciendo sonar el cascabel de los sentimientos ascendentes desde la educación; 3. Alegato humanista en favor de una compasión alta de moral; 4. Conclusión. La educación moral humanista no puede ser sustituida por la tecnología transhumanista; 5 . Referencias bibliográficas.

Cómo citar: Gracia-Calandín, J. (2020) "Transhumanismo y neuroeducación en perspectiva orteguiana", en Logos. Anales del Seminario de Metafísica 53, 55-64.

\footnotetext{
1 Esta publicación ha recibido el apoyo del proyecto de Investigación Científica y Desarrollo PID2019109078RB-C22 financiado por el Ministerio de Ciencia, Innovación y Universidades

2 Departamento de Filosofía Universitat de València javier.gracia@uv.es
} 


\section{Introducción. Neuroeducación y transhumanismo, temas de nuestro tiempo}

El mejoramiento humano ha sido un tema de reflexión constante a lo largo de la historia de la humanidad y lo es también en los tiempos actuales. ${ }^{3}$ La educación ha sido considerada la forma tradicional de mejoramiento humano y sin lugar a duda la extraordinaria capacidad para aprender y para la formación de un carácter particular a partir de la propia cultura constituye uno de los rasgos más característicos de la condición humana. Recientemente esta tesis ha cobrado fuerza a tenor de los descubrimientos en neurociencias acerca de la extraordinaria plasticidad del cerebro humano, que hace que dicho órgano sea dinámico y cambiante a lo largo de toda la vida y se conforme en interacción con el entorno educativo, cultural, histórico y social. $^{4}$

En los últimos tiempos, sin embargo, alentada por el progreso en los campos de investigación de la inteligencia artificial, la nanotecnología, la robótica o la neurociencia ha venido ganando predicamento la propuesta transhumanista, que afirma que es posible transformar sustancialmente a los seres humanos mediante la aplicación directa de la tecnología. Abundan las promesas de que la tecnología será capaz de mejorar la capacidad cognitiva de las personas mediante fármacos, la manipulación genética o la inteligencia artificial. Algunos neurocientíficos transhumanistas, por ejemplo, abogan por la implantación de aparatos que permitan a las personas optimizar sus capacidades. Otros, incluso, guiados por el negocio de las promesas transhumanistas, abogan por una biología sintética que permita rebasar la propia condición humana y alcanzar incluso la inmortalidad. Con ello se pretende eliminar la frontera entre el ser humano y la máquina y dar paso a un ser humano nuevo; por supuesto ya no sería humano; acaso ni tan siquiera "superhumano". Más bien se trataría de dar paso al "transhumano".

En este trabajo se plantea la pregunta de si es posible y deseable hablar de neuroeducación transhumanista. ¿Es la tecnología la que permitirá que las personas tengan más capacidad para el aprendizaje?, ¿o más bien la aplicación directa de la tecnología conducirá a la superación de toda pedagogía tal y como ha sido entendida hasta ahora dando lugar a una antropotécnica? ¿Implicará esa transformación técnica del ser humano un elemento a tener en cuenta en la neuroeducación o implicará el fin de toda neuroeducación?

3 Ortega, C., Richart, A., Páramo, V., Ruiz, C.: El mejoramiento humano. avances, investigaciones y reflexiones éticas y políticas, Granada, Comares, 2015.

4 Howard-Jones, P.: Evolution of learning brain. Or how you got to be so Smart, London/New York: Routledge, 2018.

5 Acuñado por Julian Huxley en los años veinte del siglo pasado como la propuesta de que "la humanidad se transcienda a sí misma”, el transhumanismo se desmarca del humanismo, que confía en la educación y la cultura para mejorar la naturaleza humana, y apuesta por aplicar la tecnología para superar los límites impuestos por nuestra herencia biológica y sintética. Véase, por ejemplo, More, M. y Vita-More, N.: The Transhumanist Reader: Classical and Contemporary Essay on the Science, Technology, and Philosophy of the Human Future, Chichester: John Wiley and Sons, 2013. Es muy interesante destacar que tras el transhumanismo y sus deseos por eliminar los límites de la naturaleza se esconden discursos tecnoutópicos cuyas consecuencias para el mundo natural pueden ser devastadoras. Es paradójico y chocante que incluso buena parte de las propuestas que tratan de recuperar y acentuar los aspectos salvajes de un área o de nosotros mismos (rewilding, en inglés), por ejemplo, mediante la biología sintética, están auspiciados, a su vez, por discursos tecnoutópicos (de Cozar, 2019) 


\section{Hipertrofia de la técnica y crisis de los deseos}

Un punto de partida para tomar el pulso a nuestra sociedad es lo que Zygmunt Bauman ha llamado la "modernidad líquida" y que consiste en la carencia de objetivos definidos y permanentes y la pérdida de lealtades que se sustituyen por un estado permanente de vigilancia. En el mundo moderno líquido, la lealtad no es motivo de orgullo sino de vergüenza y el compromiso es visto como una carga que acaba eliminando la libertad de los individuos.

En el mundo de la modernidad líquida, la solidez de las cosas, como ocurre con la solidez de los vínculos humanos, se interpreta como una amenaza [...] La perspectiva de cargar con una responsabilidad de por vida se desdeña como algo repulsivo y alarmante. ${ }^{6}$

Antonio Diéguez 7 basándose en Bauman vincula el fenómeno de la "crisis de los deseos" con la llamada "obsolescencia programada" y el deseo insaciable de nuevos productos tecnológicos. La vida útil de los productos es limitada y a pesar de los avances tecnológicos (o más bien a causa del acelerado avance de éstos) los aparatos se vuelven obsoletos más rápidamente. Sea o no programado y deliberado, de lo que no cabe duda es de que en el mundo actual marcado por el impacto social de las nuevas y cambiantes tecnologías, la vida útil de los aparatos es cada vez más corta.

La paradoja de todo ello es que el creciente deseo de disponer de los últimos y más sofisticados aparatos tecnológicos ha conducido a una acuciante "crisis de los deseos". La confianza ciega en las posibilidades de las nuevas tecnologías ha acabado por alimentar progresivamente la dependencia de dichos aparatos y, sobre todo, ha llevado a cegar las fuentes de la motivación. ${ }^{8}$ Hecho especialmente acuciante en el ámbito de la educación y que como vamos a ver genera problemas de aprendizaje.

Este es un fenómeno extraordinariamente complejo y con muchas aristas que conviene no simplificar como si de modas pasajeras se tratara. Si se quiere emplear el término por su parentesco con el de "modernidad", habría que decir que más bien es la "moda" misma la que se ha convertido en modo mutante de vida; lo provisional se ha instalado como lo permanente, dando lugar a cierta innovofilía o novolatría moderna que funde y confunde lo "nuevo" con lo "bueno". Pero conviene denunciar esta falacia de los tiempos modernos, sobre todo, cuando hablamos de innovación educativa. ${ }^{9}$ Este complejo fenómeno que alcanza el ámbito de la educación se podría caracterizar como "desmoralización educativa". ${ }^{10}$

Tal como destaca Antonio Diéguez es muy significativo que ya Ortega en su célebre Meditación de la técnica de los años treinta del siglo pasado advirtiera del peligro de la "hipertrofia de la técnica" y los efectos de ésta en términos de crisis de los deseos.

Bauman, Z.: Los retos de la educación en la modernidad líquida. Barcelona, Gedisa, 2007, p. 28.

Diéguez, A.: Transhumanismo. La búsqueda tecnológica del mejoramiento humano. Barcelona, Herder, 2017, cap. 4.

8 "Vivimos en un mundo dominado por los managers del deseo, los secuestradores de la voluntad, diseñadores y dueños de nuestras fantasías, empeñados en la creación del consumidor necesitado de una plétora de productos tecnológicos para satisfacer los deseos más banales y superficiales" (Mermall, T.: "Ortega contra Pero Grullo: Estrategias retóricas en 'Meditación de la técnica", Revista internacional de Tecnología, Conocimiento y Sociedad, 2012, vol. 1, nº 1, p. 6).

9 Gracia-Calandín, J.: "Repensando la innovación educativa en y desde la filosofía", Quaderns de Filosofia, vol. 4 (1), 2017, pp. 11-23.

10 Gracia-Calandín, J.: El desafío ético de la educación, Madrid, Dykinson, 2018, cap. 10. 
El ubérrimo elenco de posibilidades de realización que la técnica ha puesto a nuestro alcance no ha ido acompañado de un reforzamiento de la capacidad de desear o, dicho de otro modo, de la capacidad de saber elegir qué hacer con la técnica y cómo elaborar con su ayuda un proyecto vital auténtico que es el que debe darle contenido y finalidad a la propia técnica (Diéguez, 2017: 178). ${ }^{11}$

El tema de la motivación es también uno de los temas principales de los que se ha ocupado la neuroeducación. En su celebrado informe de 2007, La comprensión del cerebro. El nacimiento de una ciencia del aprendizaje, ${ }^{12}$ el grupo de renombrados especialistas en neuroeducación encargados por la OCDE incidió en la importancia de las emociones para estimular y promover cualquier tipo de aprendizaje. La neurociencia permitiría detectar qué partes del sistema límbico se activan y son claves en todo proceso de aprendizaje.

El problema de fondo no es el de si educando y educador se encuentran más o menos motivados para emprender nuevos aprendizajes, sino algo más radicalmente conectado con el temple vital de las personas, especialmente descollante en estadios tempranos del desarrollo de la personalidad. La crisis de los deseos por causa de la hipertrofia de la técnica tiene su origen en una forma de sociedad que ha dado la espalda a la incipiente y zozobrante naturaleza del infante y ha podado los mimbres a partir de los cuales ha de ir forjando su propio proyecto vital.

A la luz de contrastados estudios, conviene tener muy presentes las consecuencias perjudiciales de la sobreestimulación fomentada por aparatos tecnológicos. La hiperconectividad y el exceso de estímulos que llevan aparejados el uso de dichos aparatos, lejos de ser una herramienta para hacer niños más listos, conllevan problemas para el aprendizaje en términos de atención, autocontrol y motivación. Generan lo que en neurociencia educacional se conoce como procesos en los que el niño depende de la experiencia externa para desarrollarse (experience-dependant, en inglés) y deja de buscar activamente la experiencia (experience-expectant). ${ }^{13}$

Estos estudios concluyen que expuestos como están al bombardeo externo de estímulos con el visionado de determinados dibujos televisivos, videojuegos o aplicaciones de tabletas electrónicas, no es de extrañar que los niños se aburran, se impacienten o se pongan nerviosos cuando vuelven al ritmo del mundo real. Se asocian problemas de atención y de impulsividad con el consumo excesivo de pantallas en la infancia y la adolescencia. ${ }^{14}$ A nuestro modo de ver, la clave y el motor del proceso educativo no yace en la técnica sino en entender la naturaleza propia del ser humano para permitir su adecuado desarrollo

11 Diéguez, A.: Transhumanismo. La búsqueda tecnológica del mejoramiento humano. Barcelona, Herder, 2017. Un interesante análisis de este certero diagnóstico de José Ortega y Gasset puede encontrarse en Mitcham, C.: "La transformación tecnológica de la cultura y la crisis de los deseos", Revista de Occidente 228, 2000 pp. 33-52 y Mermall, T.: "Ortega contra Pero Grullo: Estrategias retóricas en 'Meditación de la técnica", Revista internacional de Tecnología, Conocimiento y Sociedad, 2012, vol. 1, n 1, pp. 1-10.

12 OCDE: La comprensión del cerebro. El nacimiento de una ciencia del aprendizaje. Madrid, Santillana, 2007.

13 Prestigiosos investigadores como Dimitri Christakis, director del Centro para la Salud, el Comportamiento y el Desarrollo Infantil de los EE. UU., elabora un documentado estudio sobre los nocivos efectos del visionado de dibujos animados de secuencias rápidas. Cf. Christakis, D.: "The effect of Fast-Pace Cartoons", Pediatrics, 2011, 772-774.

14 Swing, E. L., Gentile, D.A., Anderson, C.A. y Walsh, D.A. "Television and Video Game Exposure and the Development of Attention Problems", Pediatrics, 126, 2010, pp. 214-221; Zimmerman, F. J. y Christakis, D.A. "Associations between content types of early media exposure and subsequent attentional problems", Pediatrics, 120, n 5, 2007, pp. 986-992. 


\subsection{Haciendo sonar el cascabel de los sentimientos ascendentes desde la educación}

Acertadamente Antonio Diéguez señala que "se revela como imprescindible una tarea educativa que combine la adquisición de valores cívicos con la enseñanza del importante papel histórico que la ciencia y la tecnología han tenido en el desarrollo de la cultura". ${ }^{15}$ Siendo esto necesario, sin embargo, Diéguez no se detiene a profundizar en algo que es si cabe más básico y fundamental, a saber, una labor educativa que avive los deseos ascendentes y que sitúe en el centro el valor vital como potencialidad psíquica creadora. Y para ello, aunque él no alude explícitamente al ensayo de 1921 intitulado "Biología y pedagogía" del propio José Ortega y Gasset, a mi juicio, constituye un referente fundamental.

En "Biología y pedagogía" Ortega argumenta contra una falsa imagen mecanicista de la biología de impronta darwinista que entiende la vida como adaptación al medio. Esta equivocada imagen de la vida da lugar a una "pedagogía de adaptación", movida por un utilitarismo miope que poda en el niño la fronda del deseo, según el criterio del adulto. Frente a esta "biología de la adaptación", Ortega propone una "interpretación de la vida inversa de la tradición" de la que resulta una "pedagogía de secreciones internas". No es la adaptación al medio lo que prima sino que la raíz misma de la vida, el germen de vitalidad, radica en la secreción interna, la "hormona", lo "incitante". ${ }^{16} \mathrm{Su}$ interés principal es destacar que más allá de las aplicaciones médicas también hay una "vitalidad psíquica" y el objetivo es penetrar alma adentro y hallar los estratos más profundos de la vida psíquica. Es en ellos donde encontramos una "fauna psíquica inadaptada, mucho más rica, enérgica y abundante que la prudente y útil". ${ }^{17}$

Para el tema que aquí nos ocupa que vincula la hipertrofia de la técnica y la crisis humana de los deseos, es ciertamente muy iluminadora la confrontación que Ortega establece entre el "nuevo rico" que no sabe qué querer, le falta originalidad en las adquisiciones y compra lo que otros querrían, y el niño creador que muestra su salud anímica y vital en una radical fluencia psíquica y aunque irrealizable o impracticable el niño no deja de soñar y avivar su imaginación. El "nuevo rico" está marcado por una debilidad de la secreción psíquica interna del deseo y es ciertamente "pobre" en deseos. Por el contrario, el que es capaz de nutrir el querer con los deseos y alimenta los sentimientos propios de una vida ascendente, ese es el auténtico rico, rico en deseos y alto de moral.

Podría pensarse que también hay propuestas transhumanistas que conciben un humano mejorado (o incluso transhumano o hasta posthumano) a través de psicofármacos. Ortega no aborda explícitamente esta cuestión, sin embargo, su imagen del cascabel sí da pie a pensar que la intimidad que agita al niño para desear con intensidad rebasa el recurso a una técnica transhumanista (como puede ser la biología sintética). Su pedagogía del cascabel invita a educar no para la vida ya hecha sino para la vida creadora desde estas pulsiones vitales entrañadas en la condición humana, pues lo que está en juego no son estados de euforia o alteración provocados extrínsecamente, sino funciones espontáneas como el coraje, la curiosidad, el

\footnotetext{
15 Diéguez, A., Op. Cit., p. 180.

16 Cabe decir que Ortega es un muy buen conocedor de los avances científicos de su época y del surgimiento de la endocrinología y de hecho cita a Ernst Starling y a Gregorio Marañón.

17 Ortega y Gasset, J.. Obras completas, vol. 2 Madrid: Taurus y Fundación Ortega y Gasset, 2004, p. 413.
} 
amor, la agilidad intelectual, el afán de gozar y triunfar, la confianza en sí y en el mundo, la imaginación y la memoria... ${ }^{18}$ Y conviene no olvidar que todos estos son aspectos que se vinculan directamente tanto con el propio proyecto vital como con el pasado humano que de modo desbordante está ya en el niño. No es algo que haya que añadir desde fuera mediante sofisticados avances tecnológicos, sino que se trata precisamente de lo que ya está en germen en toda persona en tanto que lleva dentro a un infante.

Este díscolo personaje interior es el que nos hace tal vez reír en medio de un duelo, o decir una impertinencia a un grave magistrado, o seguir tomando el sol cuando el deber nos obliga a ausentarnos. Somos todos, en varia medida, como el cascabel, criaturas dobles, con una coraza externa, que aprisiona un núcleo íntimo, siempre agitado y vivaz. Y es el caso que, como el cascabel, lo mejor de nosotros está en el son que hace el niño interior al dar un brinco para libertarse y chocar con las paredes inexorables de su prisión. ${ }^{19}$

A la luz de esta sugerente propuesta pedagógica cabría cuestionarse si a tenor de la hiperinflación de diagnósticos de Trastornos de Déficit de Atención con Hiperactividad (TDAH) no estamos empleando la industria farmacológica para anular la vitalidad del educando y mermar sus capacidades. ${ }^{20}$ La medicalización de los niños bajo el pretexto de hiperactividad, lejos de permitir avivar la imaginación, conduce a un adormecimiento de sus capacidades. ${ }^{21}$ Por si fuera poco, este adormecimiento del "núcleo íntimo" se agrava, a su vez, con la sobreestimulación externa a la que los niños se ven expuestos con tantos aparatos electrónicos como tabletas, videojuegos, televisión... que acaban por eliminar la capacidad de asombro del niño. ${ }^{22}$

Pero incluso podríamos incidir en un problema más radical consistente en el "deseo de la gente corriente de medicalizar en lo posible su conducta y, de ese modo, reducir su grado de responsabilidad sobre sus propios actos" ${ }^{23}$ ¿Dónde queda el empoderamiento y la autonomía de la persona cuando la tecnología farmacológica se ha convertido en la clave de la conducta humana? ¿Realmente se soluciona el problema de la crisis de los deseos abogando por la tecnología? Ciertamente los intereses del marketing farmacológicos no ayudan a sondear los posibles riesgos éticos y educativos de una sociedad medicalizada. Mas el problema transhumanista no se reduce a la ingesta de fármacos más o menos generalizada, sino a la incapacidad de la persona para reconocer su propia naturaleza como propia. Por eso, incluso desde el ámbito sanitario y teniendo en cuenta los efectos iatrogénicos de los fármacos, más que escuchar al fármaco lo que hay que hacer es escuchar al paciente implementando terapias humanistas. ${ }^{24}$

18 En un interesante comentario de Manuel García Morente al ensayo de Ortega que aquí nos ocupa, afirma que "la pedagogía es la técnica de los sentimientos como propulsores de la vitalidad psíquica. La labor del pedagogo deberá consistir en provocar en el niño las reacciones sentimentales de mayor valor vital -lado positivo-, y en extirpar o impedir las emociones de menor valor vital, las emociones decadentes -lado negativo-_" (García Morente, M.: Obras Completas, Madrid, Anthropos, 1996, pp. 704-705).

19 Ortega, op. Cit., p. 424-425.

20 Frances, A.: ¿Somos todos enfermos mentales? Manifiesto contra los abusos de la psiquiatría, Barcelona, Ariel, 2014.

21 Gracia-Calandín, J. “El mejoramiento humano a través de los psicofármacos?”, en Ortega, C. y otros (eds.) El mejoramiento humano, Granada, Comares, 2015, pp. 218-227.

22 L'Écuyer, C.: Educar para el asombro, Plataforma editorial, Barcelona, 2012.

23 Fukuyama, F.: El fin del hombre. Consecuencias de la revolución biotecnológica, Madrid, BSA, 2003, p. 95.

24 González, H. y Pérez, M.: La invención de los trastornos mentales. ¿Escuchando al fármaco o al paciente? Madrid: Alianza Editorial, 2007. El problema no radica en la medicalización cuando existe una enfermedad e 
De modo análogo, ante la crisis de los deseos generada por la tecnología, es crucial que desde la educación se implementen modelos pedagógicos que devuelvan todo el protagonismo a los educandos y contribuyan al empoderamiento de sus capacidades morales y, en definitiva, el cultivo de su humanidad. ${ }^{25}$ Para ello la compasión bien entendida, la compasión alta de moral, ha de constituir una prioridad de toda educación. ${ }^{26} \mathrm{Y}$ es, por ejemplo, este aspecto que está en el centro del humanismo como consecuencia de la condición finita y vulnerable del ser humano, que la literatura transhumanista centrada en la promesa de inmortalidad del transhumano tiende a omitir.

\section{Alegato humanista en favor de una compasión alta de moral}

En "Biología y Pedagogía" Ortega pone todo el énfasis en el "pulso psíquico" (propulsor de sentimientos ascendentes) y en determinar la primacía del valor vital en su propuesta pedagógica. Una pedagogía que no se establece sobre la base de la biología evolucionista y adaptacionista iniciada por Darwin. La pedagogía del cascabel que Ortega propone no enclaustrar la vida en una adaptación al medio, ni lo vital a leyes mecánicas de actuación. Pues, repitámoslo, lo más urgente no es educar para la vida ya hecha sino para la vida creadora. Es por lo tanto un movimiento transformador de dentro hacia fuera.

En algunos pasajes Ortega parece dar a entender que la valoración ética sería algo posterior al valor vital. Que primero hay que atender a la salud vital y posteriormente a la ética. Que lo bueno biológicamente hablando difiere de lo bueno moralmente hablando. ${ }^{27}$ Sin embargo, cabría preguntarse si Ortega no está siendo en este pasaje en particular excesivamente restrictivo con el término "ética" y si realmente es posible hablar de "pura biología" en el ser humano. ¿No conduce su propio y célebre concepto de "desmoralización" a vincular lo moral con lo vital?

Por mi parte, creo que uno de los principales desafíos de la educación es reconocer su fundamento ético y descubrir que la ética no es un aditivo que se suma externamente a las pulsiones vitales, sino que la educación ética es lo que mantiene a la persona "alta de moral", y de modo complementario permite que la educación esté en buena forma y no degenere en adoctrinamiento o mera instrucción. En la

incluso un peligro real, diagnosticado y prescrito por el facultativo. Sin duda que en ocasiones la ingesta de medicamentos prescritos puede ser un factor que ayude a la autonomía del paciente. El problema filosófico de fondo, no obstante, es confundir, por ejemplo, el deseo del amor con el afán de poseer a otra persona. Para este tipo de distinciones filosóficas la farmacología es estéril y reducir el amor a una explicación psicobiológica resulta un reduccionismo excesivamente simplificador. Un ejemplo de este reduccionismo biotecnologicista puede verse en Earp, B. D.; Wudarczyk, O A.; Sandberg, A. y Savulescu, J., "If I Could Just Stop Loving You: Anti-Love Biotechnology and the Ethics of a Chemical Breakup", American Journal of Bioethics, 13 (11), pp. 3-17.

25 Calvo, P. y Gracia-Calandín, J.: Moral Neuroeducation for a Democratic and Pluralistic Society, Zurich, Springer, 2020.

26 Véase Nussbaum, M. Sin ánimo de lucro. Por qué la democracia necesita de las humanidades, Madrid, Katz, 2010 y Cortina, A.: Ética de la razón cordial, Oviedo, Nobel, 2007. Por mi parte, he argumentado prolijamente sobre esta cuestión en Gracia, J.: El desafio ético de la educación, Madrid, Dykinson, 2018.

27 "No hay que esperar la valoración ética de estos dos tipos de pulso vital. Antes que hable la ética, tiene derecho a hablar la pura biología. Sin salir de ella, desde el punto de vista estrictamente vital, nos parece el uno como un valor biológico positivo, como vitalmente bueno; el otro, como un valor biológico negativo vitalmente malo. Luego vendrá la ética y habrá lugar para discutir si lo moralmente bueno y lo moralmente malo coinciden o no con esos otros valores vitales" (Ortega, op. cit., p. 417). 
medida que se centra en la formación de un carácter, el desarrollo de la personalidad y la forja del propio proyecto vital en base a determinados valores cívicos como la libertad, el respeto, la igualdad o la justicia, la ética constituye el fin que da sentido a la labor educativa.

La desmoralización implica no solo incapacidad para actuar de modo excelente sino también incapacidad para discernir lo correcto de lo incorrecto. Humanamente hablando no tiene sentido hablar de "pura biología" sino más bien de sentimientos que nos permiten estar en buena forma y mantener la moral alta. Y por "mantener la moral alta" hay que entender tanto una mayor fluencia psíquica que le permita a la persona estar en su propio quicio y vital eficacia como el poderío para realizar conductas auténticas conforme a los propios valores morales de vida. Por ello, más que hablar de la "pura biología" o lo "estrictamente vital", desde la neuroeducación moral convendría hablar de sentimientos morales como la compasión alta de moral, que son vitales para la salud vital y moral de las personas.

La compasión alta de moral no hay que confundirla con sentir lástima por las desgracias ajenas, ni tampoco como una forma de lamento paralizante, ambos estos últimos expresión de una moral por los suelos y en ocasiones hasta de cierta performance (inauténtica sobreactuación). En tanto que pasión ética, la compasión alta de moral activa el reconocimiento de la condición vulnerable y frágil constitutiva del ser humano y de modo ejemplar conecta con la dimensión existencial del ser humano, que es irreductible a las máquinas o a la tecnología. ${ }^{28}$ Es precisamente la emergente narrativa vital (biografía) de esta autocomprensión existencial la que forja una identidad humana a la luz de la educación recibida y la singularidad del individuo en cuestión. ${ }^{29}$

¿Acaso podrían las máquinas sentir esta compasión alta de moral que nos humaniza a las personas? No lo creo. Sentir como propio el sufrimiento ajeno, sobre todo, el de los más vulnerables y con-moverse para trabajar en aras de la justicia, no solo no es un atributo transhumano, sino la clave humanista para un auténtico mejoramiento humano desde la educación. Una educación que nunca ha de dejar de reconocer nuestra frágil, finita y vulnerable condición humana. La clave no está en huir de nuestra condición humana sino en reconocerla y descubrir desde ella todo su potencial de plenitud. ${ }^{30}$

\section{Conclusión. La educación moral humanista no puede ser sustituida por la tecnología transhumanista}

El transhumanismo busca la mejora tecnológica del ser humano. Pero no la mejora desde un punto de vista coyuntural sino con el objetivo de transformar la naturaleza

28 Cortina, A.: Aporofobia, el rechazo al pobre. Un desafio para la democracia. Barcelona, Paidós, 2017, cap. 8.

29 En este punto creo que es especialmente lúcido el análisis de la identidad narrativa que lleva a cabo Paul Ricoeur al distinguir la hermenéutica de la existencia del sí, que considera irrebasable la "noción del obrar y del sufrir" donde el cuerpo es una dimensión constitutiva del sí, del "sueño tecnológico" propio de los relatos de ciencia ficción. El tipo de imaginación que funciona en uno y otro caso es sustancialmente diferente. Sólo el primero es propiamente humano y humanizante. "Las ficciones literarias difieren fundamentalmente de las ficciones tecnológicas en que siguen siendo variaciones imaginativas en torno a un invariante, la condición corporal vivida como mediación existencial entre sí y el mundo" (Ricoeur, P.: Sí mismo como otro, Madrid, Siglo XXI, 1996, pp.: 149-150).

30 Gracia-Calandín, J.: El desafio ético de la educación, op. cit. 
misma del ser humano. Y entre las propuestas de transhumano tecnológicamente mejorado encontramos un amplio abanico de promesas que van desde la creación de máquinas superinteligentes y cíborgs a biomejoramientos a través de la biología sintética. Y es que a tenor de los avances (por ejemplo, eugenésicos) hoy en día podemos hablar de "la tecnología más poderosa que el ser humano haya creado". ${ }^{31}$ Un claro exponente es la síntesis de nuevos fármacos capaces de actuar como potenciadores de las facultades físicas y psíquicas. Para los transhumanistas en virtud de esta modernísima tecnología será posible superar problemas y limitaciones que han estado presentes desde antaño y que la educación no ha conseguido erradicar.

Sin embargo, conviene tener presente en primer lugar si realmente las propuestas transhumanistas constituyen proyectos realizables o más bien promesas aún difíciles de demostrar. Pero también si es la tecnología la que va a mejorar al ser humano y qué tipo de mejora será ésta.

En perspectiva orteguiana hemos analizado la clave de la dimensión moral humana vinculada a las pulsiones psíquicas y la capacidad de desear. Y en este sentido conviene preguntarse si realmente la solución para la crisis de desear, que conduce a tantos proyectos truncados de vida humana, vendrá a través de la tecnología.

Nuestra conclusión es que constituye un error pretender sustituir la educación por la tecnología y que ello no solo no mejorará la capacidad de desear y generar deseos ascendentes en el ser humano, sino que fácilmente agravará la crisis de los deseos. La dimensión moral en su vínculo con las pulsiones psicofísicas del ser humano ha de ser una de las tareas principales de la neuroeducación para evitar la acuciante desmoralización de una sociedad en la que - para decirlo con Habermas - la ciencia, la técnica y también la tecnología se han convertido en la ideología dominante de nuestro tiempo.

Frente a un transhumanismo tecnológico que anula la dimensión moral educativa en el ser humano, en este trabajo abogamos por un humanismo en perspectiva orteguiana que ponga en el centro la capacidad de desear y que avive desde la educación, especialmente en el niño, la imaginación ética y los deseos vitalmente ascendentes.

\section{Referencias bibliográficas}

Bauman, Z. (2007). Los retos de la educación en la modernidad líquida, Barcelona, Gedisa. Calvo, P. y Gracia-Calandín, J.: Moral Neuroeducation for a Democratic and Pluralistic Society, Springer, 2020.

Christakis, D. "The effect of Fast-Pace Cartoons", Pediatrics, 2011, vol 128, n 4, 772-774.

Cortina, A.: Ética de la razón cordial. Educar en la ciudadanía en el siglo XXI, Oviedo, Nobel, 2007.

Cortina, A.: Aporofobia, el rechazo al pobre. Un desafio para la democracia, Barcelona, Paidós, 2017.

De Cózar, J.M.: "Haciendo más salvaje el antropoceno. Rewilding y domesticación”, Athenea Digital 19 (1).

31 Rosen, C.: "Build a Pet Dinosaur or Your Perfect Child", Slate, 10 de diciembre, 2014. http://www.slate.com/ articles/technology/future_tense/2014/12/synthetic_biology_advocates_are_veering_too_close_to_eugenics. single.html. Consultado el 29/08/2019. 
Diéguez, A.: Transhumanismo. La búsqueda tecnológica del mejoramiento humano, Barcelona, Herder, 2017.

Earp, B. D.; Wudarczyk, O A.; Sandberg, A. y Savulescu, J.: "If I Could Just Stop Loving You: Anti-Love Biotechnology and the Ethics of a Chemical Breakup", American Journal of Bioethics, 13 (11), pp. 3-17.

Frances, Allen. (2014). ¿Somos todos enfermos mentales? Manifiesto contra los abusos de la psiquiatría, Barcelona, Ariel.

Fukuyama, F.: El fin del hombre. Consecuencias de la revolución biotecnológica, Madrid, BSA, 2003.

García Morente, M.: Obras completas, Barcelona, Anthropos, 1996,

González, H. y Pérez, M.: La invención de los trastornos mentales. ¿Escuchando al fármaco o al paciente?, Madrid, Alianza Editorial, 2007.

Gracia-Calandín, J. “EEl mejoramiento humano a través de los psicofármacos?”, en Ortega, C. y otros (eds.): El mejoramiento humano, Granada, Comares, 2015, pp. 218-227.

Gracia-Calandín, J. "Repensando la innovación educativa en y desde la filosofía", Quaderns de Filosofia, vol. 4 (1), 2017, pp. 11-23.

Gracia-Calandín, J.: El desafío ético de la educación, Madrid, Dykinson, 2018.

Howard-Jones, P.: Evolution of learning brain. Or how you got to be so Smart, London/New York, Routledge, 2018.

L'Écuyer, C.: Educar para el asombro, Plataforma editorial, Barcelona, 2012.

Mermall, T.: "Ortega contra Pero Grullo: Estrategias retóricas en 'Meditación de la técnica", Revista internacional de Tecnología, Conocimiento y Sociedad, 2012, vol. 1, $\mathrm{n}^{\circ}$ 1, pp. $1-10$.

Mitcham, C.: "La transformación tecnológica de la cultura y la crisis de los deseos", Revista de Occidente 228, 2000 pp. 33-52.

More, M. y Vita-More, N. (2013). The Transhumanist Reader: Classical and Contemporary Essay on the Science, Technology, and Philosophy of the Human Future, Chichester, John Wiley and Sons.

Nussbaum, M. Sin fines de lucro. Por qué la democracia necesita de las humanidades. Madrid, Katz, 2010.

OCDE: La comprensión del cerebro. El nacimiento de una ciencia del aprendizaje, Madrid, Santillana, 2007.

Ortega y Gasset, José (2004-2010), Obras completas (10 vols.), Madrid, Taurus y Fundación Ortega y Gasset, .

Ortega, César; Richart, Andrés; Paramo, Víctor; Ruiz, Christian (2015). El mejoramiento humano. avances, investigaciones y reflexiones éticas y politicas, Granada, Comares.

Ricoeur, P.: Sí mismo como otro, Madrid, Siglo XXI, 1996.

Rosen, Ch. (2014), "Build a Pet Dinosaur or Your Perfect Child”, Slate, 10 de diciembre. http://www.slate.com/articles/technology/future_tense/2014/12/synthetic_biology_ advocates_are_veering_too_close_to_eugenics.single.html. Consultado el 29/08/2019.

Swing, E. L., Gentile, D.A., Anderson, C. A. y Walsh, D. A., "Television and Video Game Exposure and the Development of Attention Problems", Pediatrics, 126, 2010, pp. $214-$ 221;

Zimmerman, F. J. y Christakis, D. A., “Associations between content types of early media exposure and subsequent attentional problems", Pediatrics, 120, n 5, 2007, pp. 986-992. 